\title{
A tribute to George Sudarshan
}

\author{
S. K. Bose* \\ Department of Physics, University of Notre Dame, Notre Dame, IN 46556, USA
}

I met George Sudarshan during fall 1958, upon joining the University of Rochester, USA, as a graduate student. Sometime before that he had given a course of lectures on weak interactions at Rochester with notes taken by Jan Nilsson. These notes were now in the final stage of preparation and George asked me to help with the completion of this project, as Nilsson had already left Rochester and gone back to Sweden. I learnt much from this assignment. The V - A theory for the universal four-fermion interaction $^{1}$ was developed there with great clarity. Looking back, two situations stand out in my mind. The first is that the V-A structure was in conflict with the experimental results claimed by Rustad and Ruby ${ }^{2}$. These concern the angular correlation between the emitted electron and the neutrino in the decay of $\mathrm{He}^{6}$ to $\mathrm{Li}^{6}$. This experiment was later re-analysed and its original conclusion found to be wrong. Moreover, the corrected result was in agreement with the $\mathrm{V}-\mathrm{A}$ scheme ${ }^{3}$. Another prediction of the $\mathrm{V}-\mathrm{A}$ theory was the pion decay into electron and neutrino. More precisely, the $\mathrm{V}-\mathrm{A}$ theory predicts the branching ratio of rates $\Gamma$ :

$$
\frac{\Gamma(\pi \rightarrow e+\bar{v})}{\Gamma(\pi \rightarrow \mu+\bar{v})} \simeq(1.03) \times 10^{-4} .
$$

The above prediction was not supported by claimed experimental results of that time. The claimed results for the above ratio were an order of magnitude less than the above predicted value ${ }^{4}$. A later, apparently better, observation led to even lower value $\left(\simeq 10^{-6}\right)$ for the ratio ${ }^{5}$. However, after the publication of the $\mathrm{V}-\mathrm{A}$ theory, the electron decay mode of the pion was reinvestigated and the result now ${ }^{6}$ was in full conformity with the prediction of the $\mathrm{V}-\mathrm{A}$ theory.

One aspect of the $\mathrm{V}-\mathrm{A}$ scheme deserves more scrutiny. The chiral projection of spinor field $\psi_{ \pm}=\left(1 \pm \gamma_{5}\right) \psi$ satisfies $\gamma_{5} \psi_{ \pm}= \pm \psi_{ \pm}$. This $\gamma_{5}$ invariance is also a property of the Weyl equation (equation for a zero-mass fermion). Thinking along these lines, George got interested in studying the extreme relativistic limit (one in which the energy of the particle is much larger than the rest energy) of the Dirac equation. As such, the desired limit is quite the opposite of the non-relativistic limit found by Foldy and Wouthuysen ${ }^{7}$. George asked me to work on this problem. Upon successful completion of this project, a paper was

\footnotetext{
*e-mail: bose.1@nd.edu
}

written and published (my first $)^{8}$. A rather interesting feature of the Dirac equation in the extreme relativistic limit is that the components of the position operator do not commute. It thus anticipates the later advent of noncommutative geometry.

During the early 1960s, George became interested in the question of the exact logical basis for derivation of the space-time transformation laws; in particular, the Lorentz transformation. He and his collaborators clearly understood that the relevant transformations follow from the requirement of the invariance of the (space-time) line element and not on the invariance of the light cone (line element equated to zero). Now, the line element can be time-like, light-like, or space-like. Correspondingly, the energy-momentum 4-vector of a particle can be timelike, light-like, or space-like. For time-like 4-momentum, the particle velocity is always less than the speed of light in vacuum. For light-like 4-momentum, the particle speed is always fixed at light speed. But for space-like 4momentum, the particle speed is always greater than the speed of light. This is the genesis of the idea of fasterthan-light particles ${ }^{9,10}$. Till this proposal it was widely (albeit misleadingly) believed that light speed was the absolute upper limit to any velocity. This belief owes its origin to Einstein's 1905 paper where the relevant transformation is derived from the invariance of the light cone. Actually, the light cone has a much larger symmetry, the 15 parameter full conformal group of symmetries, of which the Lorentz group is a subgroup. This was not known in 1905, but discovered later ${ }^{11}$. Four of these additional transformations could be ruled out by somewhat arbitrarily requiring that they are realized linearly while acting on space-time coordinates. But one transformation $X^{\nu} \rightarrow \lambda X^{\nu}$ ( $\lambda$-real, non-zero) will remain. If this dilation transformation is retained, then the resultant scheme would not allow the presence of non-massless particles.

The faster-than-light particles, or tachyons, as we now call them ${ }^{12}$, have many unusual features. First, the sign of energy for a tachyon is not Lorentz-invariant. Thus, there will exist frames from which the particle will appear to possess a negative energy. The original authors ${ }^{10}$ interpreted it as a positive energy particle moving backward in time. A tachyon can exist either as a singlet or as an infinite supermultiplet whose members are distinguished by a quantum number, which can be interpreted as helicity. There are four classes of these supermultiplets.

Field theories were proposed for the singlet tachyon represented by a scalar field. The first model due to 
Tanaka $^{13}$, noted difficulties with Lorentz invariance. A Lorentz invariant unique vacuum state did not exist, nor did a unitary operator implementing a Lorentz transformation. The next attempt at constructing a field theory of scalar tachyons by Feinberg ${ }^{12}$ also encountered unusual features. The scalar field could not be quantized by canonical commutation laws; rather it required to be quantized via anti commutation relations, i.e. quantize a scalar field via Fermi rather than Bose statistics. A re-examination of the scalar tachyon field was undertaken by Arons and Sudarshan ${ }^{14}$, and fully developed by Dhar and Sudarshan ${ }^{15}$, advocating a reinterpretation of the scalar field that bypassed these difficulties. Tachyons have many other unusual properties. A tachyon falling into a Schwarzschild black hole will be stopped and turned back at a point inside the event horizon of the black hole ${ }^{16}$. Tachyons have many other theoretical problems. For a review of these, see ref. 17.

We now discuss a certain development in abstract quantum field theory (QFT) in which Sudarshan played an important part. In 1962, Jordan and Sudarshan ${ }^{18}$, Borchers $^{19}$ and Ruelle ${ }^{20}$, all independently proved the following theorem: the requirement of the uniqueness of the vacuum state (in a Hilbert space) implied that the field operators generate an irreducible ring. Borchers ${ }^{19}$ also proved the converse: irreducibility of field operators implies the uniqueness of the vacuum state. Another fundamental result proved by Araki et al. ${ }^{21}$ is that in a QFT, the uniqueness of the vacuum leads directly to the existence of the linked cluster expansion for the vacuum expectation values. Before proceeding further, we wish to mention an important point. In contrast to all the other authors cited above, who used the Wightman formulation of QFT, Jordan and Sudarshan used an algebraic formulation of QFT and made the importation observation that their result is valid in a nonrelativistic QFT as well. This is because the specific properties of Lorentz transformation were not utilized in their derivation. Similarly, Araki et $a .^{21}$ did not explicitly use Lorentz invariance (only translational invariance was used) in deriving the linked cluster expansion. Thus the result is valid in nonrelativistic QFT as well.

The first test of the aforementioned theorems came about in the Bardeen-Cooper-Schrieffer (BCS) theory of superconductivity. In the limit of infinite spatial volume, the BCS model becomes exactly soluble and was thus analysed by $\mathrm{Haag}^{22}$. He found that the ground state of the model could be described in two distinct ways. In the first description, it was characterized by a parameter $\alpha$, which took up values in a circle. For each ground state labelled by a particular $\alpha$, there was a Hilbert space $H_{\alpha}$. In each $H_{\alpha}$, the field was represented irreducibly and the linked cluster expansion was valid. In the second description, the indexed family of Hilbert spaces $\left\{H_{\alpha}\right\}$ was replaced by a single Hilbert space $H$ in which there was a string of ground states characterized by an integer. The field was reducible and the linked cluster expansion invalid in $H$. Soon afterwards appeared the paper by Araki and Woods $^{23}$ on the infinite Bose gas (with finite density). The structure of the ground states and the concomitant Hilbert spaces found here was identical with that found by $\mathrm{Haag}^{22}$. Later on, relativistic QFT models with spontaneously broken symmetries implemented via a scalar field (Higgs field) would be constructed. These models show the same structure of the vacuum states and Hilbert spaces. This is not unanticipated since the nonrelativistic models ${ }^{22,23}$ violated phase symmetry (constant gauge transformations). One remark: In any one of the Hilbert spaces $H_{\alpha}$, it is permissible to carry out QFT calculations, since the uniqueness of vacuum is one of the axioms of $\mathrm{QFT}^{24}$. On the other hand, the examples in refs 22,23 show that the reducible Hilbert space $H$ may be more suitable for physical interpretation. This is because the expectation values of gauge-dependent quantities vanish in $H$, but not in $\left\{H_{\alpha}\right\}$. Thus we have a situation in which one description is good for making field theory calculations, while the other is good for physical interpretations. This lesson should be borne in mind while considering quantum chromodynamics (see below). Finally, we should mention the following: The spaces $\left\{H_{\alpha}\right\}$ were later $^{25}$ re-interpreted as a Hilbert bundle, based on the circle; with the Hilbert space $H$ obtained via the standard construction using the Von Neumann direct integral ${ }^{26}$. Subsequently, the analysis was extended to more general gauge theories ${ }^{27}$. One conclusion of this analysis is worth mentioning. It is an interesting and unanticipated connection between spontaneous symmetry breaking and symmetric spaces. Exactly the same structure would appear in many more instances: in two-dimensional quantum electrodynamics with zero fermion mass ${ }^{28}$; in a model due to Ashtekar and $\mathrm{Sen}^{29}$, where symmetry breaking arises from the non-trivial topology of Schwarzschild-Kruskal space-time, and finally, in QCD ( $\theta$ vacuum). A conclusion from the analysis of $\mathrm{QCD}^{30}$ is noteworthy: in the reducible Hilbert space $H$, there is "no violation of $\mathrm{CP}$ invariance and therefore no need for the hypothesis of an axion'.

During the later 1960s, I became interested in a class of representations (the most degenerate representations) of the non-chiral group $\operatorname{su}(3) \otimes \mathrm{su}(3)$ in connection with the description of mesons ${ }^{31}$. The scheme was then developed in association with Sudarshan ${ }^{32}$, during which the structure of these representations became transparently clear. The representations are of the form $(\overline{3}, 3),(\overline{6}, 6)$, $(\overline{10}, 10) \ldots$ of the group $\operatorname{su}(3) \otimes \operatorname{su}(3)$. The first is Okubo's nonet, the second is suitable for a meson that consists of two antiquarks and two quarks. As for the masses, a generalized equal spacing rule was proved. The scheme would be rediscovered within the specific context of the MIT bag model later ${ }^{33}$.

No description of Sudarshan's scientific contributions can be complete without recalling his work on the statistical 
states of the radiation field. Specifically, he proposed for the density matrix operator of the field the following representation $^{34}$

$$
\rho=\int \phi(z)|z\rangle\langle z| \mathrm{d}^{2} z,
$$

In the above $|z\rangle$ is a coherent state, which is the right eigenstate of the annihilation operator, $z$ is a complex number and $\phi(z)$ is a real function (due to hermiticity of $\rho)$. The above, the so-called diagonal coherent state representation, is adequate to describe all statistical states of the radiation field. This is due to the fact, as Sudarshan noted, that the basis provided by coherent states is overcomplete. Now comes a crucial observation. Although real, the function $\phi(z)$ need not always be positivevalued. The positive values correspond to fields that are classical and negative values correspond to fields that are non-classical. This is significant. We recall that many centuries before, the intellectual ancestors of Sudarshan had figured out the significance of negative numbers: if a positive number denotes an asset, a negative one is a liability. Finally, the inverse of the above formula was also presented, as a result bringing the whole exercise to a mathematical completion. Thus was born the quantum theory of optical coherence. Later on, the Sudarshan representation would be rechristened the GlauberSudarshan P representation (and sometimes simply as the Glauber P representation)! But that is another story.

Sudarshan was also interested in the connection between spin and statistics of a particle. The spin-statistics theorem (integer spin particles obey Bose and half odd integer spins obey Fermi statistics) is considered an important development in (relativistic) QFT. Sudarshan enquired if relativistic invariance was really necessary. After all, the introduction of spin does not require the Lorentz or Poincare groups; the rotation subgroup $\mathrm{SO}(3)$ or its double cover SU(2) is enough. Similarly, the concept of statistics is meaningful for an assemblage of nonrelativistic particles; as in the electronic theory of metallic conductivity. These considerations led to the derivation of the spin-statistics theorem in a nonrelativistic quantum field theory ${ }^{35,36}$.

Sudarshan became interested in the conceptual problems connected with observations on a transient quantum system. The project was carried out in collaboration with Baidyanath Misra $^{37}$. Specifically, these authors considered an unstable particle with half-life equal to $T$. Let the particle be created at the instant $t *$. After an interval $\tau$, let the particle be subjected to an observation as regards its presence. The result will be positive if $\tau$ is very much smaller than $T(\tau \ll T)$, since the unstable particle does not get enough time to decay. Thus, the system will start its evolution all over again starting with the instant $t *+\tau$. In effect, the lifetime of the particle has been extended by $\tau$. Repeated observations will thus have an observable effect. In the limit of continuous observations, the particle will not decay and become stable. The authors called this phenomenon the quantum Zeno effect. This has been observed by Itano et al. $^{38}$.

In addition to being an outstanding scientist, Sudarshan was also a man of wide intellectual interests. One could get him interested in almost any problem under the sun. He was once interested in applying techniques of operations research to increase the efficiency of Indian railways. With the passage of time, he grew increasingly involved with the questions raised by the Vedantic philosophy. He was also a very generous man; as all those who came in contact with him will be aware.

I understand that Sudarshan was nominated for the Nobel Prize half a dozen times. But the prize eluded him. In this, he is undoubtedly in illustrious company. The list includes physicists like J. C. Bose, M. N. Saha, S. N. Bose, G. N. Ramachandran and N. S. Kapany. All this may be a matter of plain coincidence. It may also be indicative of a certain Weltanschauung that is entrenched in the western intellectual tradition.

1. Sudarshan, E. C. G. and Marshak, R. E., Report in Padua-Venice Conference (Sept 1957); Proceedings (eds Zonichelli, N. and Bologna, P.), 1958, pp. 11-14; Phys. Rev., 1958, 109, 1850; Also, Feynman, R. P. and Gell-Mann, M., Phys. Rev., 1958, 109, 193.

2. Rustad, B. M. and Ruby, S. L., Phys. Rev., 1953, 89, 880; 1955, 97, 991 .

3. Hermannsfeld, W. B. et al., Phys. Rev. Lett., 1958, 1, 61.

4. Lokanathan, S. and Steinberger, J., Suppl. Nuovo Cimento, 1955, $1,151$.

5. Anderson, H. L. and Lattes, C. M., Nuovo Cimento, 1957, 6, 1356.

6. Anderson, H. L., Fuji, T., Miller, R. H. and Tan, L., Phys. Rev., 1960, 119, 2050

7. Foldy, L. L. and Wouthuysen, S. A., Phys. Rev., 1949, 78, 29

8. Bose, S. K., Gamba, A. and Sudarshan, E. C. G., Phys. Rev., 1959, 113, 1551.

9. Bilaniuk, O. M., Despande, V. K. and Sudarshan, E. C. G., Am. J. Phys., 1962, 30, 718.

10. Bilaniuk, O. M. and Sudarshan, E. C. G., Nature, 1969, 223, 386; Phys. Today, 1969, 212, 43.

11. Bateman, H., Proc. London Math Soc, 1910, 8, 223; E. Cunningham, Proc. London Math Soc., 1910, 8, 77.

12. Feinberg, G., Phys. Rev., 1967, 159, 1089.

13. Tanaka, S., Prog. Theor. Phys (Kyoto), 1960, 24, 171

14. Arons, M. E. and Sudarshan, E. C. G., Phys. Rev., 1968, 173, 1622.

15. Dhar, J. and Sudarshan, E. C. G., Phys. Rev., 1968, 174, 1808.

16. Raychaudhuri, A., J. Math. Phys., 1974, 15, 856.

17. Bose, S. K., J. Phys: Conf. Ser., 2009, 196, 012022.

18. Jordan, T. F. and Sudarshan, E. C. G., J. Math. Phys., 1962, 3, 587.

19. Borchers, H. J., Nuovo Cimento, 1962, 24, 214.

20. Ruelle, D., Helv. Phys. Acta, 1962, 35, 167.

21. Araki, H., Hepp, K. and Ruelle, D., Helv. Phys. Acta, 1962, 35, 164.

22. Haag, R., Nuovo Cimento, 1962, 25, 187.

23. Araki, H. and Woods, E. J., J. Math. Phys., 1963, 4, 637.

24. Streater, R. F. and Wightman, A. S., PCT, Spin and Statistics, and All That, Benjamin, New York, USA, 1964, p. 98.

25. Bose, S. K., J. Math. Phys., 1980, 21, 2839. 
26. Mackey, G., Unitary Group Representations in Physics, Probability and Number Theory, Benjamin, Reading, Mass, USA, 1978.

27. Bose, S. K., Phys. Rev. D, 1981, 24, 2153; 1982, 26, 525.

28. Lowenstein, J. and Swieca, A., Ann. Phys., 1971, 68, 172.

29. Ashtekar, A. and Sen, A., J. Math. Phys., 1980, 21, 526.

30. Bose, S. K., Z. Naturforsch, 1997, 52a, 133.

31. Bose, S. K., Phys. Rev., 1965, 150, 1231; Nuovo Cimento, 1966, 46, 419 .

32. Bose, S. K. and Sudarshan, E. C. G., Phys. Rev., 1967, 152, 1396; Phys. Rev. Lett., 1989, 62, 1445.

33. Jaffe, R. J., Phys. Rev. D, 1977, 15, 267; 281.
34. Sudarshan, E. C. G., Phys. Rev. Lett., 1963, 10, 277.

35. Sudarshan, E. C. G., Proc. Indian Acad. Sci. A, 1968, 67, 284; Duck, I. M. and Sudarshan, E. C. G., Am. J. Phys., 1998, 66(4), 284.

36. Duck, I. M. and Sudarshan, E. C. G., Pauli and the Spin-Statistics Theorem, World Scientific, Singapore, 1998.

37. Misra, B. and Sudarshan, E. C. G., J. Math. Phys., 1977, 10, 756.

38. Itano, W. M., Heinzen, D. J., Bollinger, J. J. and Wineland, D. J., Phys. Rev. A, 1980, 41, 2295.

doi: $10.18520 / \mathrm{cs} / \mathrm{v} 116 / \mathrm{i} 2 / 207-210$ 